\title{
Interperiod Capacitance Calculations for Three-Dimensional Multiconductor Systems
}

\author{
RUEY-BEEI WU
}

\begin{abstract}
A novel approach is proposed to facilitate the capacitance calculations for periodic three-dimensional multiconductor systems. Based on the Fourier transform technique, the approach requires only the conductors inside one period and solves the charge distribution in the spectral domain by the integral equation method. The resultant spectral capacitances are then inverse transformed to give the capacitances between any two conductors, which may even be inside different periods. The approach is finally applied to the capacitance analysis for connector pins in a packaging board design.
\end{abstract}

\section{INTRODUCTION}

A $S$ THE SWITCHING of logic gates becomes faster, the coupling effect between densely spaced interconnections plays an ever increasing role in the timing analysis of the whole system. The capacitances between these sometimes complicated interconnections now become crucial elements in the modeling and computer-aided electric analysis of high-performance systems [1].

Except for certain simple structures where empirical or approximate formulas apply, the capacitance calculations for most practical structures resort to numerically intensive methods such as the finite-element method (FEM) [2] and the integral equation method (IEM) [3]-[7]. Of these, IEM, which is easier with respect to the modeling and solution of the charge distribution on the conductor surfaces, is more popular for three-dimensional multiconductor systems. However, its usage is rather limited since, for more complicated structures, it frequently exhausts the available computer storage or computation time.

As the scale of integration increases, practical structures exhibit a higher degree of periodicity. It becomes almost impossible for IEM to model and solve the charge distribution of the conductors in all the periods. Some approaches were proposed to alleviate this numerical difficulty by exploiting the structural periodicity [8], [9]. They require solution of only one period and thus save a great deal of computer storage and computation time. However, they can consider only the capacitances between intraperiod conductors.

When one is concerned about the capacitances between two conductors in different periods, a novel approach as

Manuscript received February 5, 1988; revised April 26, 1988. This work was supported in part by the National Science Council, Republic of China, under Grant NSC-77-0404-E002-08.

The author is with the Department of Electrical Engineering, National Taiwan University, Taipei, Taiwan, R.O.C.

IEEE Log Number 8823261.

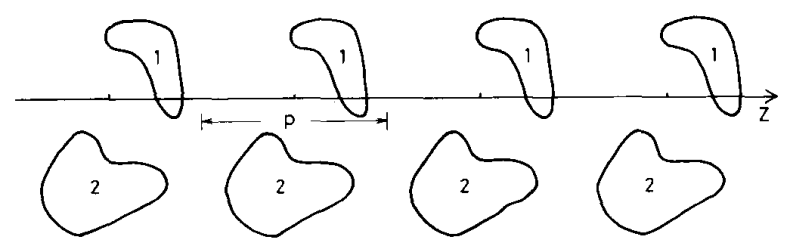

Fig. 1. A typical structure periodic in the $z$ direction with a period $p$

proposed here should be employed. The approach is based on the Fourier transform technique. In Section II, we depict the capability of calculating the interperiod capacitances by the solution of only one period in the transformed spectral domain. The numerical formulas for the associated Green's function in the spectral domain are derived in Section III. Numerical examples are then included in Section IV to demonstrate and verify the significant features of this approach. Finally, some brief discussions and conclusions are presented in Section $\mathrm{V}$.

\section{Spectral ANALYSIS ApProach}

Consider a three-dimensional multiconductor system (Fig. 1) which is periodic in the $z$ direction with period $p$. The number of conductors in one period is $M$. In the notation, choose one of the periods as the base, or zeroth period. All the others are numbered as the $k$ th periods accordingly $(k= \pm 1, \pm 2, \cdots, \pm \infty)$. Let $V_{k}(n)$ and $Q_{k}(n)$ be the voltage and the total charge on the $n$th conductor in the $k$ th period. Then, they satisfy

$$
Q_{l}(m)=\sum_{n=1}^{M} \sum_{k=-\infty}^{\infty} C_{I-k}(m, n) \cdot V_{k}(n)
$$

for $m=1,2, \cdots, M$ and $l=0, \pm 1, \pm 2, \cdots, \pm \infty$. Here, $C_{l-k}(m, n)$ is the short-circuit capacitance [5] between the $m$ th conductor in the $l$ th period and the $n$th conductor in the $k$ th period. For a periodic system, the capacitance is shift invariant and thus can be indexed as $l-k$.

Solution of $C_{l-k}(m, n)$ calls for the conductor charges in all the periods. A better alternative is to take the Fourier transform of (1) and solve the problem in the spectral domain, i.e.,

$$
\tilde{Q}(m ; f)=\sum_{n=1}^{M} \tilde{C}(m, n ; f) \cdot \tilde{V}(n ; f) .
$$

Here and throughout this paper, a quantity with a tilde 
denotes its Fourier transform, i.e.,

$$
\begin{array}{r}
\tilde{Q}(m, f)=\mathscr{F}\left[Q_{k}(m)\right]=\sum_{k=-\infty}^{\infty} Q_{k}(m) \cdot e^{j 2 \pi k f} \\
(0 \leqslant f<1) .
\end{array}
$$

The solution of spectral capacitance $\tilde{C}(m, n ; f)$ in (2) is much easier. One needs only to know how to model and solve the charge distribution for the $M$ conductors in the base period. After finding $\tilde{C}(m, n ; f)$ for every frequency $0 \leqslant f<1$, the desired capacitance $C_{k}(m, n)$ can be obtained by the inverse Fourier transform:

$$
\begin{aligned}
C_{k}(m, n)=\int_{0}^{1} \tilde{C}(m, n ; f) \cdot e^{-j 2 \pi k f} d f \\
\\
\quad(k=0, \pm 1, \pm 2, \ldots, \pm \infty) .
\end{aligned}
$$

Physically, the value $C_{k}(m, n)$ equals the total charge induced on the $m$ th conductor in the $k$ th period when a unit voltage is imposed on the $n$th conductor in the base period while all other conductors are grounded.

Actually, only the values of $C(m, n ; f)$ for $0 \leqslant f \leqslant 1 / 2$ are necessary since the spectral capacitance satisfies $\tilde{C}(m, n ; 1-f)=[\tilde{C}(m, n ; f)]^{*}$, where * denotes the complex conjugate. The integral in (4) can be evaluated numerically by many methods, such as the Simpson rule and the fast Fourier transform.

\section{Spectral GreEn's Function}

The spectral capacitances can be found by an integral equation method. The procedure is identical to the conventional one [4] except that a suitable Green's function in the spectral domain should be employed. Let the surface of the $n$th conductor in the base period be denoted by $S_{n}$ $(n=1,2, \cdots, M)$. Then, the conductor charge density $\tilde{q}(r ; f)$ in the spectral domain satisfies the integral equation

$$
\sum_{m=1}^{M} \int_{S_{m}} \tilde{G}\left(\boldsymbol{r}, \boldsymbol{r}^{\prime} ; f\right) \cdot \tilde{q}\left(\boldsymbol{r}^{\prime} ; f\right) d \boldsymbol{r}^{\prime}=\tilde{V}(n ; f)
$$

for $r \in S_{n}(n=1,2, \cdots, M)$.

Here, the spectral Green's function $\tilde{G}\left(\boldsymbol{r}, \boldsymbol{r}^{\prime} ; f\right)$ is the Fourier transform of

$$
G_{k}\left(\boldsymbol{r}, \boldsymbol{r}^{\prime}\right)=G\left(\boldsymbol{r}+k p \hat{z}, \boldsymbol{r}^{\prime}\right)
$$

where $G\left(\boldsymbol{r}, \boldsymbol{r}^{\prime}\right)$ is the conventional Green's function [4] and $\hat{z}$ is a unit vector in the $z$ direction. When all these conductors are in a homogeneous medium of dielectric constant $\epsilon$,

$$
G\left(r, r^{\prime}\right)=\left[4 \pi \epsilon\left|r-r^{\prime}\right|\right]^{-1}
$$

and thus

$$
\begin{aligned}
\tilde{G}\left(\boldsymbol{r}, \boldsymbol{r}^{\prime} ; f\right)= & \frac{1}{4 \pi \boldsymbol{\epsilon}} \cdot\left\{\frac{1}{\left|\boldsymbol{r}-\boldsymbol{r}^{\prime}\right|}\right. \\
& \left.+\sum_{k=1}^{\infty}\left[\frac{e^{j 2 \pi k f}}{\left|\boldsymbol{r}+k p \hat{z}-\boldsymbol{r}^{\prime}\right|}+\frac{e^{-j 2 \pi k f}}{\left|\boldsymbol{r}-k p \hat{z}-\boldsymbol{r}^{\prime}\right|}\right]\right\} .
\end{aligned}
$$

For structures with several layered dielectric regions, the
Green's function is much more complicated. Nevertheless, the simplifications proposed here can be applied in similar fashion.

Just as in the conventional approach [4], [9], (5) can be reduced to a matrix equation by using a suitable discretization procedure and applying the Galerkin's method [9]. However, the associated matrix here is a complex Hermitian matrix since the spectral Green's function satisfies $\tilde{G}\left(\boldsymbol{r}, \boldsymbol{r}^{\prime} ; f\right)=\left[\tilde{G}\left(\boldsymbol{r}^{\prime}, \boldsymbol{r} ; f\right)\right]^{*}$.

Assigning a unit voltage to the $n$th conductor while all others are grounded $(n=1,2, \cdots, N$ sequentially), the unknown charge density $\tilde{q}(\boldsymbol{r} ; f)$ can be solved from the complex matrix equation. The total charge on the $m$ th conductor,

$$
\tilde{Q}(m ; f)=\int_{S_{m}} \tilde{q}(\boldsymbol{r} ; f) d \boldsymbol{r} \quad(m=1,2, \cdots, M)
$$

and thus the spectral conductance $\tilde{C}(m, n ; f)$ can be obtained accordingly. Here, the values of $\tilde{C}(m, n ; f)$ are in general complex numbers.

A successful implementation of the approach relies on an efficient evaluation of the spectral Green's function $\tilde{G}\left(\boldsymbol{r}, \boldsymbol{r}^{\prime} ; f\right)$. When the frequency $f$ is zero, the series in (6) diverges. An additional constraint of charge neutrality together with the associated regularization algorithm has been proposed to find the corresponding capacitances [9]. In other cases for which $0<f<1 / 2$, the series converges but very slowly. Some numerical simplifications are proposed to facilitate the calculations.

By Taylor's expansion with respect to $1 / k$, the Green's function $G_{k}\left(\boldsymbol{r}, \boldsymbol{r}^{\prime}\right)$ for $k \neq 0$ is decomposed into two parts, i.e.,

$$
\begin{aligned}
4 \pi \epsilon G_{k}\left(\boldsymbol{r}, \boldsymbol{r}^{\prime}\right)= & \left|\boldsymbol{r}+k p \hat{z}-\boldsymbol{r}^{\prime}\right|^{-1} \\
= & \frac{1}{|k p|} \cdot\left(1-\frac{z}{k p}+\frac{z^{2}-\rho^{2} / 2}{(k p)^{2}}-\frac{6 z^{3}-3 z \rho^{2} / 2}{(k p)^{3}}\right) \\
& +R_{k}\left(\boldsymbol{r}-\boldsymbol{r}^{\prime}\right)
\end{aligned}
$$

where $z=\left(\boldsymbol{r}-\boldsymbol{r}^{\prime}\right) \cdot \hat{z}, \rho^{2}=\left[\left(\boldsymbol{r}-\boldsymbol{r}^{\prime}\right) \cdot \hat{x}\right]^{2}+\left[\left(\boldsymbol{r}-\boldsymbol{r}^{\prime}\right) \cdot \hat{y}\right]^{2}$, and $R_{k}\left(r-r^{\prime}\right)$ is the remainder. Substituting (7) into (6), the spectral Green's function can thus be calculated by evaluating the series sums of the two parts in (7) respectively.

The slowly convergent behavior of the bracket in (6) is now reflected in the series $\sum_{k=1}^{\infty} k^{-l} e^{j 2 \pi f}(l=1,2,3,4)$, which are contributed from the first part in (7). These series depend on the frequency $f$ but are independent of the coordinates $\boldsymbol{r}$ and $\boldsymbol{r}^{\prime}$. As listed in the Appendix, some analytical formulas can be employed for an efficient evaluation.

For the series of the second part in (7), no analytical formulas are available and one should resort to the numerical summation. However the series converges very fast due to the $1 / k^{5}$ dependence of $R_{k}$ and $R_{-k}$ for large 


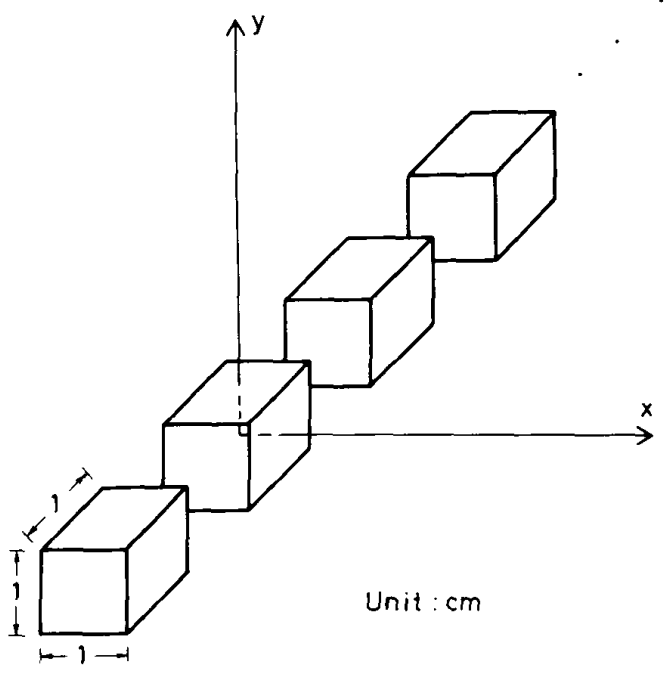

Fig. 2. A simple periodic structure consisting of a cubic conductor array.

$k$. Usually, ten summation terms from $k=1$ to 10 can compute the result to a relative error smaller than $10^{-4}$.

\section{Numerical EXAMPLES}

A general-purpose capacitance calculation program which can fully utilize the structural symmetry and periodicity, if any, has been proposed for three-dimensional multiconductor systems [9]. With the aforementioned algorithm embedded, the program now can calculate the capacitances in periodic structures even if the two conductors of interest are not inside the same period.

Generally, the algorithm proposed here should be employed when the interperiod conductors are not connected together. In applying the algorithm, the program first calculates the spectral capacitances $\tilde{C}(m, n ; f)$ for $2^{L}+1$ frequencies $\left(f=l / 2^{L+1}, l=0,1,2, \cdots, 2^{L}\right)$, where $L$ is a user-specified integer. Then, (4) is applied to approximate $C_{k}(m, n)$, i.e., the capacitance between the $n$th conductor in the base period and the $m$ th conductor in the $k$ th period. The results range for $m=1,2, \cdots, M ; n=$ $1,2, \cdots, M$; and $k=0, \pm 1, \pm 2, \cdots, \pm 2^{L}$.

One method for evaluating the integral in (4) is the inverse fast Fourier transform (FFT). Due to the aliasing error inherent in FFT, it can be proved that the result obtained will be an overestimation for the mutual capacitance but an underestimation for the self-capacitance. The other integration method is the Simpson rule, which, in general, gives more accurate results. However, it is difficult to predict whether the result obtained will be an overestimation or underestimation. Intuitively, satisfactory results are guaranteed when both methods give close solutions. Otherwise the integer $L$ should be increased to achieve more consistent results.

To demonstrate the proposed theory, consider a very simple periodic structure consisting of a cubic conductor array as shown in Fig. 2. In the numerical solution, each side of the cubic is discretized into eight segments. Since

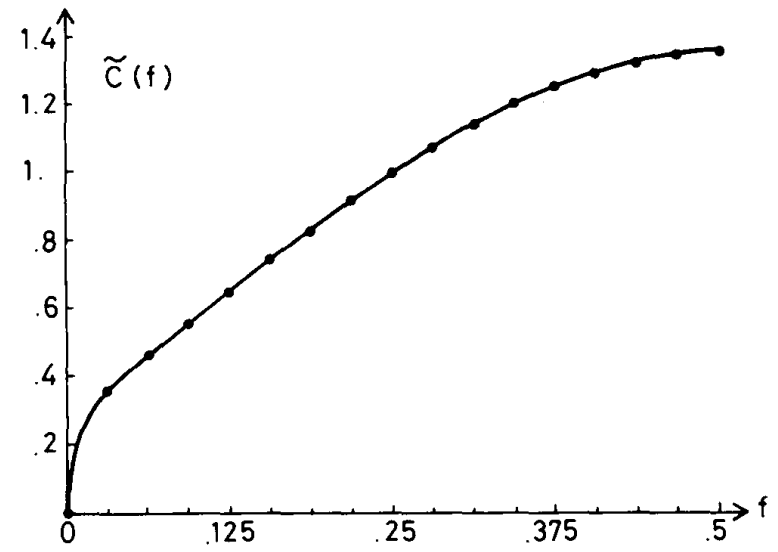

Fig. 3. Spectral capacitance $\tilde{C}(f)$ versus frequency $f$ for the cubic conductor array shown in Fig. 2.

the cubic conductor is even-symmetric with respect to both the $x=0$ and $y=0$ planes, only the conductor surfaces in the region $x \geqslant 0$ and $y \geqslant 0$ are actually called for [9]. The number of unknowns is thus $(8 \times 4+4 \times 4) \times 2=96$. This can be compared with the number $8 \times 8 \times 6=384$ when one does not utilize the structural symmetry. Also, it should be noted that the structural symmetry in the $z$ direction cannot be exploited here since the asymmetric factor $e^{j 2 \pi k f}(f \neq 0)$ imposed on the conductor charge distribution destroys the symmetry between the $k$ th and $(-k)$ th periods.

The spectral capacitance $\tilde{C}(f)$ can be obtained by solving the integral equation (5). Shown in Fig. 3 is the real part of $\tilde{C}(f)$ versus the frequency $f$. The imaginary part of $\tilde{C}(f)$ is zero since this structure is symmetric with respect to the $z=0$ plane. By (4), the spectral capacitance can be used to find the desired result $C_{k}$, which is the capacitance between the two conductors: one in the base period and the other in the $k$ th period.

For example, let us choose the integer $L=4$. The program calculates 17 spectral capacitances as denoted by the dots in Fig. 3. Both the FFT and the Simpson rule are employed to find $C_{k}$, which is equal to $C_{-k}$ in this example. The results for $k=0,1,2, \cdots, 8$ by the two methods are listed in Table I. It is found that the self-capacitance $C_{0}$, with a lower bound of $0.92727 \mathrm{pF}$ (by inverse FFT), is approximately $0.93040 \mathrm{pF}$ (by Simpson's integration). On the other hand, the mutual capacitance $\left|C_{1}\right|$, with an upper bound of $0.24285 \mathrm{pF}$ (by the inverse FFT), is about $0.23976 \mathrm{pF}$ (by Simpson's integration). Similar results hold for other $C_{k}$ 's.

Without resorting to this spectral analysis approach, one can also consider several periods together to approximate the interperiod capacitances. Of course, all the conductors in these periods should be modeled and solved simultaneously. The desired capacitances can be obtained directly. However, this benefit is offset by the requirement of a large number of unknowns, and thus large computer storage and computation time. For example, let us include seven periods in the calculation and adopt the same discre- 
TABLE I

Capacitance $C_{k}$ Inside the Cubic Conductor ArRay Shown in Fig. 2: COMPARISON OF THREE METHODS

\begin{tabular}{|c|c|c|c|}
\hline$k^{\text {method }}$ & inverse FFT & Simpson rule & Direct \\
\hline 0 & 0.92727 & 0.93040 & 0.93206 \\
\hline 1 & -0.24285 & -0.23976 & -0.23820 \\
\hline 2 & -0.04715 & -0.04416 & -0.04314 \\
\hline 3 & -0.02676 & -0.02393 & -0.02747 \\
\hline 4 & -0.01940 & -0.01675 & $\times$ \\
\hline 5 & -0.01577 & -0.01331 & $\times$ \\
\hline 6 & -0.01368 & -0.01138 & $\times$ \\
\hline 7 & -0.01235 & -0.01016 & $\times$ \\
\hline 8 & -0.01145 & -0.00928 & $\times$ \\
\hline
\end{tabular}

Results with $k$ ranging from 0 to 8 are in $\mathrm{pF}$.

tization. If structure symmetry is not exploited, the number of unknowns for this simple structure is 2688 , almost impossible to solve! Even fully utilizing the structural symmetry, this number is 336 , still much larger than 96 .

The results by this direct approach are also listed in Table I for comparison. Since only seven periods are included, only the capacitances between the central conductor and the next three conductors are available. These results are reliable except for $C_{3}$, which exceeds the upper bound of $0.02676 \mathrm{pF}$. This discrepancy may be attributed to the absence of the shielding effect from the fourth period. It is thus inferred that one should at least include the structure up to the $(k+1)$ st period to give reliable $C_{k}(m, n)$.

The second example considers the capacitance analysis for connector pins which are designed in the board to contact the card. Several tens of pins are periodically aligned in the $z$ direction with pitch $p=2.54 \mathrm{~mm}$. In each period, there are four pins symmetrically distributed to match both sides of the card so as to increase the connectivity. The geometry for the two connector pins in the right-hand side of the card is shown in Fig. 4.

By assuming the structure is periodic, the program applies the spectral analysis approach and calls for four conductors in the base period. Moreover, due to the structural symmetry with respect to the $x=0$ plane, only the two conductors, as shown in Fig. 4, are actually modeled and solved. Then, the spectral capacitances between the four conductors in the base period can be obtained by the modal decomposition technique [9]. In the numerical solution, each pin is divided into three segments, as shown by the dotted curves in the figure. Each segment is of cylindrical shape but is modeled as a hexagon. With the discretization along the segment axis as depicted in the figure, the total number of unknowns is 228 .

Let the two conductors in Fig. 4 be indexed as conductors $\# 1$ and $\# 2$, while their image counterparts in the $x<0$ region are $\# 3$ and $\# 4$. By choosing the integer $L=4$ and solving the spectral capacitances, the short-circuit capacitances $C_{k}(m, n)$ 's are found from (4) by the Simpson rule. The numerical results for $k=0,1,2,3$ are listed in Table II. The number in the parenthesis is the

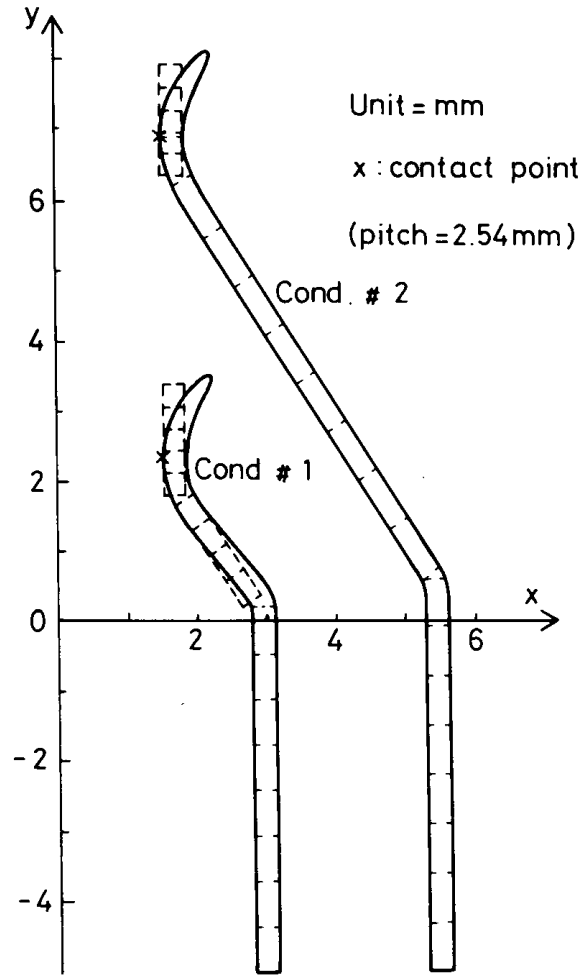

Fig. 4. Geometry for connector pins in the board.

TABLE II

Capacitance $C_{k}(m, n)$ for the Periodic Structure Consisting of CONNECTOR Pins Shown IN Fig. 4

\begin{tabular}{|c|c|c|c|c|}
\hline$(m, n)$ & $C_{0}(m, n)$ & $c_{1}(m, n)$ & $C_{2}(m, n)$ & $C_{3}(m, n)$ \\
\hline $\begin{array}{l}(1,1) \\
(3,3)\end{array}$ & .16137 & $-.01102(.069)$ & $-.00150(.009)$ & $-.00045(.003)$ \\
\hline $\begin{array}{l}(2,2) \\
(4,4)\end{array}$ & .23494 & $-.02517(.107)$ & $-.00570(.024)$ & $-.00246(.010)$ \\
\hline$\frac{(1,2)}{(3,4)}$ & $-.04526(.232)$ & $-.01069(.055)$ & $-.00220(.011)$ & $-.00089(.005)$ \\
\hline (1.3) & $-.01293(.080)$ & $-.00515(.032)$ & $-.00112(.007)$ & $-.00037(.002)$ \\
\hline $\begin{array}{l}(1,4) \\
(2,3)\end{array}$ & $-.00663(.034)$ & $-.00365(.019)$ & $-.00124(.006)$ & $-.00059(.003)$ \\
\hline$(2,4)$ & $-.01150(.049)$ & $-.00571(.024)$ & $-.00227(.010)$ & $-.00127(.005)$ \\
\hline
\end{tabular}

Results with $k=0,1,2,3$ are listed here in $\mathrm{pF}$. Numbers in parentheses are coupling coefficients.

capacitance coupling coefficient, which is defined by

$$
K_{k}(m, n)=-C_{k}(m, n) / \sqrt{C_{0}(m, m) \cdot C_{0}(n, n)} \text {. }
$$

As listed in Table II, the self-capacitance, which is of primary interest in pin design, is $0.23494 \mathrm{pF}$ for the longer pin and $0.16137 \mathrm{pF}$ for the shorter pin. Another important parameter is the coupling coefficient, which should be small enough to avoid significant signal crosstalk. For the 
pin design in this example, there is a very large coupling ( $K=0.232$ ) between conductors $\# 1$ and $\# 2$, or $\# 3$ and $\# 4$, in the same period. Also, the coupling between adjacent $\# 2$ or $\# 4$ conductors is large $(K=0.107)$. These closely coupled conductor pairs deserve serious consideration in the design of high-performance systems.

\section{Discussions AND CONCLUSIONS}

The interperiod capacitance calculations for periodic three-dimensional multiconductor systems are investigated in this paper. One may employ a direct approach by including enough periods and solving the charge distribution for all the conductors inside these periods. Instead, this paper proposes a spectral analysis approach which handles this problem in an indirect but efficient manner. This approach requires the conductors in only one period and solves the spectral capacitances repeatedly for several frequencies. These spectral capacitances are then inverse Fourier transformed to obtain the desired interperiod capacitances.

Usually, the periodic structure encountered in a practical system consists of a large number of periods. The direct approach solves the capacitances by assuming a finite, usually very small, number of periods, while the indirect approach assumes an infinite number of periods. Both approaches achieve consistent results for the conductors inside periods close together. However, the direct approach is usually abandoned due to its requirement of a large number of unknowns and thus large computer storage and computation time. In this respect, the indirect approach, which requires much less computational capacity, should be more advantageous for complicated structures.

\section{APPENDIX}

\section{NUMERICAL FormUlas FOR SPECTRAL} GREEN's Function

By substituting (7) into (6), the spectral Green's function becomes

$$
\begin{array}{r}
\tilde{G}\left(\boldsymbol{r}, \boldsymbol{r}^{\prime} ; f\right)=\frac{1}{4 \pi \epsilon} \cdot\left\{\frac{1}{\left|\boldsymbol{r}-\boldsymbol{r}^{\prime}\right|}+A_{1} \frac{2}{p}-j A_{2} \frac{2 z}{p^{2}}+A_{3} \frac{2 z^{2}-\rho^{2}}{p^{3}}\right. \\
-j A_{4} \frac{12 z^{3}-3 z \rho^{2}}{p^{4}}+\sum_{k=1}^{\infty}\left[R_{k}\left(\boldsymbol{r}-\boldsymbol{r}^{\prime}\right) e^{j 2 \pi k f}\right. \\
\left.\left.+R_{-k}\left(\boldsymbol{r}-\boldsymbol{r}^{\prime}\right) e^{-j 2 \pi k f}\right]\right\}
\end{array}
$$

where the constants are

$$
\begin{array}{ll}
A_{1}=\sum_{k=1}^{\infty} \frac{\cos 2 \pi k f}{k} & A_{2}=\sum_{k=1}^{\infty} \frac{\sin 2 \pi k f}{k^{2}} \\
A_{3}=\sum_{k=1}^{\infty} \frac{\cos 2 \pi k f}{k^{3}} & A_{4}=\sum_{k=1}^{\infty} \frac{\sin 2 \pi k f}{k^{4}} .
\end{array}
$$

These can be calculated by the following formulas:

$$
\begin{aligned}
A_{1}= & -\ln (2|\sin \pi f|) \\
A_{2}= & 2\left(A_{1}+1\right) u-\frac{2 u^{3}}{9}-\frac{2 u^{5}}{225}-\frac{4 u^{7}}{6615}-\frac{2 u^{9}}{42525} \\
& -\frac{4 u^{11}}{1029105}-\cdots \\
A_{3}= & \zeta_{3}-2\left(A_{1}+\frac{3}{2}\right) u^{2}+\frac{5 u^{4}}{18}+\frac{7 u^{6}}{675}+\frac{u^{8}}{1470} \\
& +\frac{11 u^{10}}{212625}+\frac{13 u^{12}}{3087315}+\cdots \\
A_{4}= & 2 \zeta_{3} u-\frac{4}{3}\left(A_{1}+\frac{11}{6}\right) u^{3}+\frac{u^{5}}{5}+\frac{34 u^{7}}{4725}+\frac{83 u^{9}}{178605} \\
& +\frac{82 u^{11}}{2338875}+\frac{38 u^{13}}{13378365}+\cdots
\end{aligned}
$$

where $u=\pi f$ and $\zeta_{3}=1.2020563 \cdots$. The expressions for $A_{2}, A_{3}$, and $A_{4}$ are satisfactory when $f$ is small, say $0<f \leqslant 0.3$. For larger $f$, say $0.3<f \leqslant 1 / 2$, better expressions are

$$
\begin{aligned}
A_{2}= & -2 A_{1} v+\frac{2 v^{3}}{3}+\frac{2 v^{5}}{15}+\frac{4 v^{7}}{105}+\frac{34 v^{9}}{2835}+\frac{124 v^{11}}{31185}+\cdots \\
A_{3}= & -\frac{3}{4} \zeta_{3}-2 A_{1} v^{2}+\frac{5 v^{4}}{6}+\frac{7 v^{6}}{45}+\frac{3 v^{8}}{70}+\frac{187 v^{10}}{14175} \\
& +\frac{403 v^{12}}{93555}+\cdots \\
A_{4}= & \frac{3}{2} \zeta_{3} v+\frac{4}{3} A_{1} v^{3}-\frac{3 v^{5}}{5}-\frac{34 v^{7}}{315}-\frac{83 v^{9}}{2835}-\frac{1394 v^{11}}{155925} \\
& -\frac{1178 v^{13}}{405405}-\cdots
\end{aligned}
$$

where $v=\pi(1 / 2-f)$.

\section{REFERENCES}

[1] A. E. Ruehli, "Survey of computer-aided electrical analysis of integrated circuit interconnection," IBM J. Res. Develop., vol. 23, pp. 626-639, Nov. 1979.

[2] P. E. Cottrell and E. M. Buturla, "VLSI wiring capacitance," IBM J. Res. Develop., vol. 29, pp. 277-288, May 1985 .

[3] P. D. Patel, "Calculation of capacitance coefficients for a system of irregular finite conductors on a dielectric sheet," IEEE Trans. Microwave Theory Tech., vol. MTT-19, pp. 862-869, Nov. 1971.

[4] A. E. Ruehli and P. A. Brennan, "Efficient capacitance calculations for three-dimensional multiconductor system," IEEE Trans. Microwave Theory Tech., vol. MTT-21, pp. 76-82, Feb. 1973.

[5] A. E. Ruehli and P. A. Brennan, "Capacitance models for integrated circuit metallization wires," IEEE J. Solid-State Circuits, vol. SC-10, pp. 530-536, Dec. 1975.

[6] S. M. Rao, A. W. Glisson, D. R. Wilton, and B. S. Vidula, "A simple numerical solution procedure for statics problems involving arbitrary-shaped surfaces," IEEE Trans. Antennas Propagat., vol. AP-27, pp. 604-608, Sept. 1979 
[7] S. M. Rao, T. K. Sarker, and R. F. Harrington, "The electrostatic field of conducting bodies in multiple dielectric media," IEEE Trans. Microwave Theory Tech., vol. MTT-32, pp. 1441-1448, Nov. 1984.

[8] H. C. Lee and H. D. Chai, "Integral point-matching method for two-dimensional Laplace field problems with periodical boundaries," IBM J. Res. Develop., vol. 24, pp. 622-630, Sept. 1980.

[9] R. B. Wu and L. L. Wu, "Exploiting structure symmetry and periodicity in capacitance calculations for three-dimensional multiconductor systems," IEEE Trans. Microwave Theory Tech., vol. 36, pp. 1311-1318, Sept. 1988.

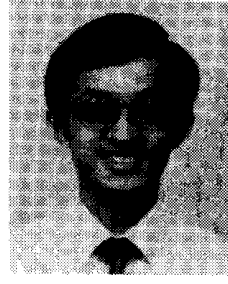

Ruey-Beei Wu was born in Tainan, Taiwan, Republic of China, on October 27, 1957. He received the B.S.E.E. degree from National Taiwan University, Taipei, Taiwan, in 1979, and the $\mathrm{Ph} . \mathrm{D}$. degree from the same university in 1985

In 1982 , he joined the faculty of the Department of Electrical Engineering, National Taiwan University, where he is now an Associate Professor. In 1986, he was a Visiting Scientist for one year at the IBM General Technology Division Laboratory, East Fishkill Facility, Hopewell Junction, NY. His research interests include numerical techniques for electromagnetics and electrical analysis of computer packaging. 